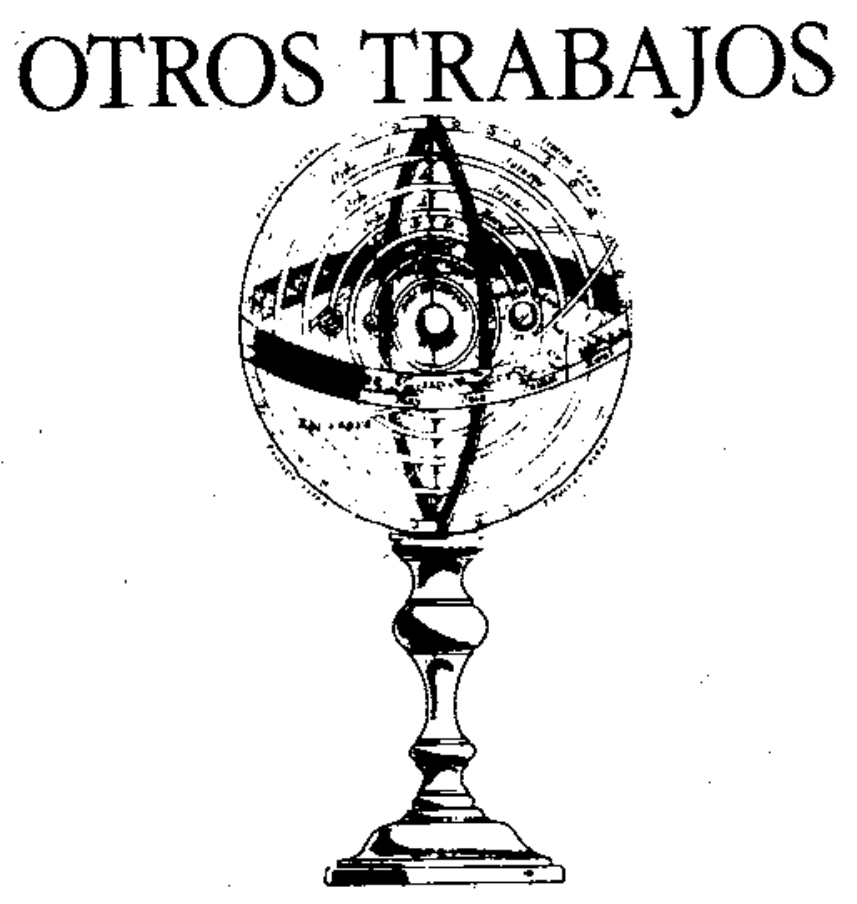

\title{
LA EDUCACIÓN PARA LA SALUD: UNA PROPUESTA FUNDAMENTADA DESDE EL CAMPO DE LA DOCENCIA
}

GAVIDIA CATALÁN, V.1-2, RODES SALA, M.J.² y CARRATALÁ BEGUER, A.²

${ }^{t}$ Departamento de Didáctica de las Ciencias Experimentales. Universitat de València.

${ }^{2}$ Programa Educación para la Salud. Conselleria de Cultura, Educació i Ciència. València.

\section{SUMMARY}

Is Sanitary Education the same as Health Education? Wich factors should we keep in mind when setting up a curriculum for Health Education? Is it necessary to deal with such health matters as hygiene, vacunations and so on... in order to develop this type of education for schools? These are just some of the questions that are raised in this article, and at some time we show the use of looking at sources that determine a curriculum, as in the specific case of Food-Nutrition.

\section{LA ROSA ES SIEMPRE LA ROSA, PERO EXIS-}

TEN DIFERENCIAS ENTRE EDUCACION PARA LA SALUD Y EDUCACIÓN SANITARIA

Los conceptos y las prácticas de salud pública se conocen desde los tiempos más antiguos. Los egipcios poseían canales para desagüar las aguas residuales y aljibes para almacenar el agua potable. A los hebreos, el Levítico 
considerado el primer código de higiene escrito (1500 años $\mathrm{aC}$ ) les indicaba que debían mantener limpio su cuerpo, desinfectar las viviendas después de una enfermedad, proteger el agua y los alimentos de contaminación, aislar a los leprosos, etc.

Sin embargo, la salud pública desde la perspectiva actual de fílosofía social, práctica administrativa y política de gobierno, se desarrolla tan sólo desde mediados del siglo pasado, gracias a Chadwick (1842) en Inglaterra y a Shattuck (1850) en EEUU, Establecen con claridad la relación entre pobreza y enfermedad y demuestran que la miseria reinante en los barrios periféricos de las ciudades industriales, donde se instalan la suciedad, el desarraigo y la marginación, son la causa de la elevada tasa de mortalidad y baja esperanza de vida.

Todo ello motivó la creación de los primeros Servicios de salud pública bajo la denominación de «Sanidad e Higiene Pública», con el objeto de proteger la salud de la población mediante acciones de gobierno dirigidas al medio ambiente y a la colectividad (Rosen 1958). Este objetivo fue posíble por la aplicación de los descubrimientos bacteriológicos de Pasteur y Kock. Es Ia llamada etapa bacteriológica de la salud pública o «primera revolución epidemioiógicas (Terris 1980).

Las vacunas y los sueros controlaron las enfermedades de esta época, pero también la ingeniería y arquitectura sanitarias tuvieron gran importancia, al canalizar las aguas residuales y dotar de casas más habitables a los habitantes de los barrios marginales. Se previno así el contagio de nuevas enfermedades y se promocionó la salud entre la población. De esta manera los servicios de higiene pública además de la protección de la salud de los ciudadanos, asumieron actividades de fomento y promoción, al comienzo del presente sigio (Ferrara et al. 1975)

Winslow (1920) indica que la salud pública debe procurar el saneamiento del medio, el control de las enfermedades transmisibles, la organización de los servicios médicos. Y por vez primera, se habla de educación sanitaria.

De este modo, bajo el concepto de salud pública, y como una parte necesaria de la misma, aparece la educación sanitaria. Su objetivo es educar a la población para prevenir las enfermedades y promocionar su salud, ya que de la restauración se hacen cargo los sectores de asistencia y rehabilitación. La acción educativa recae sobre el individuo, al que se le intenta instruir sobre la nocividad de ciertos comportamientos, con el objeto de que modifique su vida hacia hábitos más saludables. Es llevada a cabo por personal sanitario.

La idea de salud comunitaria aparece a mediados del presente siglo como evolución natural del concepto de salud pública (Lathem y Newberry 1970, Schwartz 1974). Se basa en la responsabilidad y participación de la sociedad en los procesos de planifícación, administración, gestión y control de las acciones que llevan al óptimoestado de salud de sus integrantes. Aunque continúa interviniendo el Gobierno, la comunidad, a través de sus asociaciones, tiene un protagonismo desde el mismo momento de la decisión y diseño de las acciones en el campo de la salud y del control de lo realizado.

Desarrolla el concepto de educación para la salud (EpS) para el cumplimiento de sus objetivos, de la misma forma que la saluđ pública necesitó la educación sanitaria de la población (Salleras 1985). La diferencia entre ambas radica en dos aspectos:

- Su origen. La educación sanitaria nace del concepto de salud pública y la educación para la salud, del de salud comunitaria, más evolucionado, con la carga de participación y auto-responsabilidad que ello conlleva.

- Su concepción. La EpS contempla al individuo no como un elemento pasivo de su intervencion, sino que éste participa activamente en el incremento y mejora de su propia salud. Admite que la conducta humana no sólo está determinada por decisiones propias y factores internos individuales, sino también, por elementos externos ambientales, de manera que ante determinados condicionantes exógenos, difícilmente habrá modificaciónes de laconducta individual. De esta manera, toda la comunidad debe intervenir en la educación para la salud.

Como consecuencia de la sensibilización creada por la misma, se produce una autoresponsabilidad en el cuidado y promoción de la salud, que puede llegar a una modificación de actitudes, y en algunos casos de comportamientos, hacia estilos de vida más saludables.

La educación para la salud informa, motiva y ayuda a la población a adoptar y mantener prácticas y estilos de vida saludables, propugna los cambios ambientales necesarios para facilitar estos objetivos y dirige la formación profesional y la investigación hacia estas metas. En opinión de la OMS (1983), la EpS es «cualquier combinación de actividades de información y educación que lleve a una situación en la que la gente desee estar sana, sepa como alcanzar la salud, haga lo que pueda individual y colectivamente para mantener la salud y busque ayuda cuando la necesite».

Los cambios de valores en la civilización occidental, en cuanto a justicia social, democracia y participación, bacen que las prácticas de salud pública se vayan convirtiendo en acciones de salud comunitaria (Law 1980), de la misma forma que la educación sanitaria se va confundiendo en educación para la salud, aunque hemos visto que los conceptos son distintos y el camino que media entre ambos es largo.

Superado el discurso divergente de ambos conceptos, pensamos que es mejor propiciar su confluencia. Los dos términos deben expresar el mismo objetivo: mejorar la salud individual y colectiva, incidiendo no sólo desde y con el individuo, sino también con el entorno. Y en este sentido, podemos decir con Becaud, que «la rosa es siempre la rosan.

La EpS ya no es responsabilidad única de la Sanidad, sino que son muchos los sectores de la sociedad que están implicados. Entre todos elíos destaca de manera 
obvia el mundo de la educación, Io que ha sido entendido desde siempre por el profesorado, que ha incorporado a su docencia diaria prácticas y contenidos de salud (Linville 1909).

\section{CONDICIQNES PARA UNA ACCIÓN EN EDUCACION PARA LA SALUD (EpS)}

La educación para la salud es un método de trabajo en la actividad de loseducadores y de los profesionales sanitarios. Según Modolo (1979) «es un instrumento que ayuda aI individuo a adquirir un conocimiento científico sobre problemas y comportamientos útiles par alcanzar el objetivo «salud».

La OMS en 1954 resume en tres los objetivos de la Educación para la Salud:

1. Obtener que el concepto de salud se inserte en Ios «valores» reconocidos por la comunidad.

2. Ofrecer a la población conocimientos suficientes y capacidad práctica para resolver los problemas de salư.

3. Favorecer el desarrollo de los servicios sanitarios.

Para conseguir estos objetivos, Modolo señala que toda acción de educación para la salud debe reunir los requisitos y consideraciones siguientes:

1. La educación para la salud no debe centrarse sólo en el individuo, debe comprender al grupo, ya que los comportamientos individuales tienen en gran medida una influencia grupal, social.

2. Se deben abordar problemas concretos, relevantes y percibidos. No se deben tratar problemas no sentidos, pues carecen de significación, ni tampoco generales, que por ser excesivamente amplios, difuminan una posible actuación. Es importante recoger las vivencias de los individuos.

3. La acción debe ser continua y no puede quedarse en actuaciones puntuales, esporádicas o de «campañas». La formación de actitudes y comportamientos requiere un tiempo superior al necesario para la mera información.

4. Se debe tener en cuenta la competencia de los individuos. Cada persona tiene unos comportamientos y actitudes debido a una historia y a una serie de condicionantes que elia misma conoce y valora, por lo que decidirá cambiarlos o no en función de su escala de valores.

5. El profesional de la educación para la salud no debe establecer una relación de autoritarismo, presentando un mensaje de forma dogmática como «la verdad», sino que debe presentarse como colaborador en la búsqueda de soluciones a problemas de interés para todos.

6. La información suministrada debe ser veraz e íntegra, es decir, no debe ser parcial, falsa o deformada. Recono- ciendo la dificultad de la neutralidad, se debe buscar anular las tendencias por confrontación de los datos provenientes de diversas fuentes.

7. Se debe procurar la motivación necesaria para que los individuos acepten los mensajes, superando los obstáculos y resistencias culturales y psicológicas hacia determinados problemas, ayudándolos a poner en práctica las soluciones encontradas.

\section{EL CONSTRUCTIVISMO COMO TEORÍA PSICOLOGICA DE LA ENSENANZA- APRENDIZAJE}

Constructivismo es un término que se refiere a cómo aprendemos, es decir, «a la forma en que tanto los individuos como los grupos construyen ideas acerca de cómo funciona el mundo» (Novack 1988). Es opuesto al positivismo o empirismo, en el sentido de que éste sostiene que el conocimiento es universal y permanece, por lo que el sentido del aprendizaje radica en descubrir este conocimiento verdadero.

Durante mucho tiempo, la teoría dominante en la enseñanza era la conductista o behaviorista, y uno de sus mejores autores fue B.F. Skinner. Se creía que la enseñanza consistía en presentar un adecuado estímulo $(E)$ al alumno, de forma que éste genera una determinada respuesta $(R)$, y con la repetición en el tiempo se produce un enlace $E-R$, de forma que cuando aparece $E$, inmediatamente surge $R$. En esto consiste la enseñanza, en seleccionar convenientemente los $\mathrm{E}$ que generen las $\mathrm{R}$.

No obstante, se advirtió que las propuestas que presentaba el educador no siempre significaban un $\mathrm{E}$ para todos los alumnos, y tampoco todos los alumnos producían el tipo de respuesta que esperaban los profesores. A ello hay que añadir que los binomios E-R formados tampoco eran estables, desapareciendo en el tiempo. El conductivismo no explicaba cómo aprendían los humanos y esto llevó al desarrollo de las ideas cognoscitivas del aprendizaje.

Ausubel (1978) pone su énfasis en el aprendizaje significativo en contraste con el aprendizaje por repetición. Esta significación debe ser lógica y psicológica: lógica por estar de acuerdo con la estructura interna de la disciplina a tratar; psicológica, por que debe estar de acuerdo con los conocimientos previos de quien aprende. "Averíguese lo que el alumno sabe y actúese en consecuencia.»

Todo esto conlleva una serie de puntos que caracterizan Io que podemos denominar la perspectiva constructivista del aprendizaje, entre los que, según Driver (86) podemos destacar:

1. El alumno es el protagonista de su propio aprendizaje, ya que es él quien construye sus conocimientos. El profesor debe elaborar las estrategias necesarias y crear las circunstancias precisas para que el alumno aprenda. 
2. Se deben tener en cuenta los conocimientos previos de Ios alumnos, que se estructuran en forma de esquemas con los que operan.

3. El aprendizaje consiste en la modificación de los esquemas mentales de los alumnos por incorporación de nuevos conceptos, lo que equivale a un aumento de su complejidad, o bien por modificación en las relaciones que se habían establecido entre los conceptos.

4. Los temas a tratar deben ser relevantes para los alumnos, favoreciendo la relación entre ciencia, técnica y sociedad.

5. Las fuentes de aprendizaje son muchas y variadas, no residen exclusivamente en el profesor, revelándose los compañeros de los alumnos como un factor de aprendizaje a contemplar y potenciar. De ahí, la importancia de los trabajos en grupo, la emisión de hipótesis, el diseño de experiencias, la evaluación de resultados, etc.

6. La necesidad de construir los conocimientos, supone una inversión de tiempo mayor que la simple transmisión de conceptos. Esto significa la reestructuración de los contenidos de las programaciones.

La propuesta actual es la de seleccionar adecuadamente unos contenidos que constituyan un núcleo fundamental y coherente para profundizar de forma paulatina, y a partir de ahí realizar las necesarias ampliaciones. Es decir, confeccionar un currículo en espiral, de forma que lo fundamental se trate en actuaciones sucesivas y cada vez con mayor profundidad.

7. El aprendizaje significativo conlleva una interiorización de actitudes y una aceptación de nuevos valores, por lo que es necesario desarrollar actitudes positivas hacia el objeto del aprendizaje.

\section{EL CONSTRUCTIVISMO EN EDUCACIÓN PARA LA SALUD, ¿UNA CORRIENTE DE MODA O UNA NECESIDAD METODO- LOGICA?}

EI estilo de vida de las personas, aspecto básico en la promoción de la salud, depende en gran manera de su capacidad de elegir, de tomar decisiones. Esto sólo puede desarrollarse si el currículo escolar se plantea de una forma holística, destinada a la formación integral de los alumnos y alumnas, y no simplemente a la trasmisión del conocimiento.

Establecer la relación entre los factores de riesgo y el daño para la salud requiere un adiestramiento que permita desarrollar la capacidad crítica de los alumnos sobre sus propios conocimientos. Estas ideas, diferentes en cada individuo, configuran el modo propio de vivir, de relacionar experiencias y comportamientos, de manera que es necesario iniciar las actividades de enseñanzaaprendizaje averiguando el punto de partida de cada alumno, sus ideas previas, en las cuales intervienen muchas veces tradiciones y prejuicios.
De esta forma, para fomentar el aprendizaje, no sólo de conocimientos sino también de actitudes y de habilidades, no es indiferente la metodología didáctica que se utilize. El método tradicional de trasmisión de información, de normas y pautas de comportamiento, no parece el apropiado.

Para hacer educación para la salud en la escuela, no hace faita introducirla como una nueva disciplina. Si analizamos las propuestas educativas de la perspectiva constructivista y las implicaciones metodológicas que conllevan, y las comparamos con las condiciones de una acción de Educación para la Salud que especifica Modolo, podemos liegar a la conclusión de que no existen diferencias entre ambas.

Ello nos puede inducir a pensar que todo profesor o profesora que siga un planteamiento constructivista de la enseñanza-aprendizaje, metodológicamente está haciendo educación para la salud.

Si reflexionamos sobre el concepto de salud de la OMS (1985) que indica que «es la capacidad de desarrollar el propio potencial personal y de responder de forma positiva a Ios retos del ambiente, considerando la salud, no como el objeto de la vida sino un recurso para la misma», podemos pensar que, también en cuanto a contenidos, toda acción educativa es una acción de educación para la salud, como decía Jefferson en 1875 , «la salud noes más que el saber».

El profesor que potencia la construcción de conocimientos sintoniza con el concepto de salud de la OMS, o ¿acaso no pretende conseguir el máximo desarrollo de las capacidades de su alumnado? Cuando se enseña a leer, escribir, estudiar, observar la naturaleza que nos rodea, dibujar perspectiva caballera, etc., ¿no se desarrollan las posibilidades personales?, ¿no se está haciendo educación para la salud?

Podríamos concluir que el profesorado, tanto por contenidos como por metodología, realiza una acción intrínsecamente de EpS, no siento necesario hablar de higiene bucodental, sexualidad o alimentación, para llevarla a cabo.

No obstante, nos preguntamos si Jefferson y su escuela tienen razón y, por lo tanto, la educación para la salud no es más que la educación para el saber.

\section{ELEMENTOS OUE CONFIGURAN UN CURRICULUM DE EDUCACION PARA LA SALUD}

El objetivo último de la educación es el desarrollo intelectual, afectivo y psicomotor de los alumnos, es decir, su desarrolto integral como persona, de forma que todos los contenidos que se tratan, están en función de ese último fin, y la metodología utilizada es aqueila que no sólo permite el aprendizaje de conceptos y habilidades, sino también la adquisición de los valores, normas y actitudes relacionadas con ellos. 
Éste es el enfoque con el que se hace educación para la salud, pero ¿es ésta la educación para la salud que se debe hacer en la escuela?

A decir verdad, conseguir estos ambiciosos objetivos ya sería mucho, pero desde la perspectiva de EpS no es suficiente. La escuela tiene la obligación de responder a las necesidades que la sociedad tiene planteadas, y la sociedad actual tiene una serie de problemas de salud que no pueden ser tratados única y sintomáticamente desde el campo de la medicina. Nos referimos a los problemas de cronificación de enfermedades, envejecimiento de la población, uso y abuso de drogas y fármacos, sedentarismo, malnutrición, problemas cardiovasculares, etc.

Todos estos problemas tienen en común una causa que los origina: el estilo de vida. El desarrollo de las diferentes pautas de conducta debe ser tratado en la escuela, ya que la edad escolar es el momento idóneo para ello.

La elección de los contenidos a desarrollar, conceptuales, procedimentales o actitudinales deben responder a cuatro factores distintos (Coll 86), que también denominamos fuentes, ya que de ellas parten las exigencias de su tratamiento. Son las fuentes sociológica, epistemológica, psicológica y pedagógica que determinan todo currículo (Gimeno Sacristán, J. y Pérez A. 1983). También en el currículo de Educación para la Salud se pueden establecer estas cuatro fuentes que pasamos a considerar brevemente.

Fuente Sociológica de la Educación para la Salud. Se basa en las nuevas necesidades sociales y las demandas que hace la sociedad para que ciertos temas se desarrollen en la escuela, por considerarlos necesarios para los ciudadanos del futuro.

Si la escuela es un lugar de socialización donde se transmite el patrimonio cultural y por donde pasan todos los ciudadanos en el momento de su vida en que es más fácil el aprendizaje e interiorización de ciertos conceptos, valores y normas de comportamiento, es en la escuela, donde se debe capacitar a los alumnos a analizar críticamente los valores culturales, preparándolos para una sociedad plural y diversa. Necesitamos, por ello, conocer los problemas que tiene la sociedad, para abordarlos en consecuencia.

Entre las cuestiones prioritarias actuales en relación a la salud, podemos indicar, los estilos de vida, la alimentación poco saludable, la falta de ejercicio físico, la dificultad en Ias relaciones humanas, los movimientos migratorios, la ciudades deshumanizadas, el aumento del paro, el consumismo, el envejecimiento de la pobłación, los problemas de higiene, etc. que conllevan unas enfermedades que difícilmente se curan con el tipo de medicina tradicional.

De ahí, el cambio de estrategia solicitado por la OMS (1981) cuando lanza su campaña de «Salud para todos en el año 2000 ", en la que indica que se pretende conseguir:
- La salud como norma de vida.

- La prevención de la enfermedad.

- Cuidados comunitarios para todos.

Para los dos primeros puntos se requiere un cambio de mentalidad y de conducta en los ciudadanos, que sólo se puede lograr a través de la educación.

Fuente Epistemológica de la Educación para la Salud. Se basa en la estructura intema de la disciplina a desarro. Ilar, tos conocimientos científicos, su historia y su estado actual. Establece las relaciones interdisciplinares pertinentes, diferencia lo fundamental de lo accesorio y presenta una metodología de trabajo.

En la EpS es de gran importancia el análisis de la evolución histórica de los diferentes conceptos de salud. No es ésta una cuestión anecdótica, puesto que cada concepto ha ido aportando un rasgo diferente y ha marcado el nivel de conocimiento que existe en la sociedad en cada momento, indicando un camino a recorrer para llegar a alcanzar el concepto de salud que poseemos en la actualidad. Esto significa que debemos tener en cuenta el nivel de partida, los preconceptos de salud de nuestros alumnos para, a partir de ahí, iniciar el proceso de enseñanza-aprendizaje.

Fuente Psicológica de la Educación para la Salud. Se basa en los procesos de desarrollo intelectual, procesual y psicomotor de los alumnos, las regularidades que presentan las diferentes etapas evolutivas, las diferencias individuales existentes, y los mecanismos y procesos de aprendizaje.

En educación para la salud cobra especial importancia el desarrollo de actitudes y pautas de comportamiento, que configuran un determinado estilo de vida. La psicología social nos aporta información sobre las bases científicas de Ia modificación de los comportamientos de salud. En general, en el contexto de la educación para la salud, se considera que una actitud es «una predisposición mental adquirida y duradera, que incita a comportarse de una forma determinada frente a un determinado tema de salud» (Salleras 1985).

La adopción o modificación de un determinado comportamiento sólo se puede producir si se presentan actitudes positivas hacia el mismo, aunque no siempre el cambio de actitud lleve inequívocamente a una modificación de la conducta, puesto que también los factores externos (familia, compañeros, amigos), el medio ambiente so. cial del individuo, influyen en ésta. Las actitudes son modificables por el aprendizaje, siempre que este aprendizaje sea significativo e interiorizado, de forma que llegue a cuestionar los valores y creencias.

Pero al igual que no existe un único estilo de vida saludable, ya que éste depende del entorno social y cultural en el que nos movemos, tampoco podemos basar la promoción de la salud únicamente en comportamientos individuales, sino que debemos tener un enfoque ecológico, ya que las raíces de los problemas de salud tienen factores políticos, económicos y sociales. 
Fuente Pedagógica de la educación para la salud Consiste en la intervención del profesorado en el hecho didáctico, diseñando y llevando a efecto una adecuada secuencia de enseñanza-aprendizaje. Se basa en la experiencia docente y en las investigaciones psicopedagógicas.

Las actividades planteadas y la metodología empleada deben estar en función de lo que queremos conseguir. $\mathrm{Si}$ pretendemos proporcionar destrezas para la vida, aumentar la autoestima, desarrollar la capacidad de elección, de adaptación, de comunicación, de saber gestio" nar el tiempo con efectividad, y de sercríticos y constructivos con el ambiente que nos rodea, debemos utilizar recursos, estrategias y metodología adecuadas para ello. Las simulaciones, presentación de problemas, investigaciones, juegos, caracterizaciones, debates, etc. son estrategias que, usadas adecuadamente y en el momento oportuno, dan resultados óptimos.

En este sentido y como propuesta metodológica, debemos contar con la participación de padres, sanitarios y demás agentes sociales y tener en cuenta los presupuestos enunciados por Modolo (1979) para una acción de educación para la salud. Está demostrada la dificultad de obtener algún cambio individual si no hay colaboración en instancias comunitarias que faciliten y refuercen la puesta en práctica de una nueva opción del alumno a la cual ha podido llegar por decisión personal en el trayecto de su aprendizaje escolar.

\section{APLICABILIDAD DE LAS FUENTES QUE DETERMINAN EL CURRICULO DE EDU- CACION PARA LA SALUD}

¿Para qué nos puede servir la constatación y concreción de las cuatro fuentes que determinan el currículo de educación para la salud?

En primer lugar, nos ayuda a contestar las preguntas que nos hemos formulado anteriormente, y a señalar cuándo y cómo las acciones que realicemos en la escuela deben ser contempladas como de educación para la salud. Hemos apuntado que toda acción educativa que respete los principios constructivistas, es educación para la salud, pero esto no es toda la educación para la salud que la escuela y la sociedad necesitan.

Si no se hace algo más, sólo se tienen en cuenta las fuentes psicológicas y pedagógicas de la educación para la salud, pero no las sociológicas que la sociedad demanda, ni las epistemólogicas que otorgan coherencia lógica a nuestras acciones pedagógicas. La consideración de las cuatro fuentes que determinan su currículo es indispensable para un correcto tratamiento.

En segundo lugar, la atención de hacer explícitas las fuentes curriculares no es únicamente testimonial sino que pedagógicamente es válida y de gran utilidad para el profesorado, ya que le facilita el trabajo de realizar su programación.

A título de ejemplo, podemos tomar el caso de la alimen * tación-nutrición. El hecho de considerar las cuatro fuen. tes, como muestra el esquema que se adjunta, facilita la concreción del currículo, dando sentido a las actividades que planteamos para su desarrollo.

Ante cualquier tema que consideremos de educación para la salud, debemos contestar a las preguntas planteadas desce las distintas fuentes:

- Desde la fuente sociológica: ¿Por qué es importante en la socieđad actual? ¿Qué debe conocer todo futuro ciuđadano? ¿Cómo debemos intervenir en los problemas que la sociedad tiene planteados?

- Desde la fuente epistemológica: ¿Cuáles son los conocimientos científicos actuales acerca del tema? ¿Cuál ha sido su historia y evolución? ¿Qué tendencias existen? ¿Cuál es el método de trabajo de los investigadores?

- Desde la fuente psicológica: ¿Cuál es el nivel de madurez intelectual de los alumnos con los que tratamos? ¿Qué intereses poseen y con qué valores se mueven? ¿Cuáles son sus comportamientos y estilos de vida? $i \mathrm{~A}$ qué razones obedecen?

- Desde la fuente pedagógica: ¿De qué manera pueden aprender e interiorizar ciertos conceptos, informaciones y valores? ¿Cómo conseguir desarrollar su capacidad de elección? ¿Cómo convertirlos en ciudadanos críticos con ellos mismos y con lo que le rodea? $¿$ Qué problemas e investigaciones habría que presentar que lleven al aprendizaje deseado?

Las respuestas a estas preguntas, la reflexión sobre ellas y la elaboracion de un esquema como el presentado ofrecen las pautas metodológicas de actuación del profesorado y facilitan la concreción de una programación.

\section{¿QUÉ EDUCACIÓN PARA LA SALUD DEBEMOS DESARROLLAR ENLAESCUELA? RESUMEN Y CONCLUSION}

Hemos visto, en este artículo, que no es lo mismo salud pública que salud comunitaria y las diferencias que existen entre educación sanitaria y educación para la salud. La tendencia actual es hacia esta última, que es un concepto más amplio y positivo y que debe suponer que, en toda acción educativa, adecuadamente planteada y resuelta, se hace educación para la salud.

No obstante, pensamos que ello no es suficiente, creemos que en la escuela, además de lo anteriormente considerado, se debe tratar aspectos de higiene, nutrición, sexualidad, drogadicción, SIDA, etc. Es necesario abordar los problemas de salud concretos que posea la población escolar e intentar que nuestros alumnos y alumnas desarrollen pautas de conducta y estilos de vida saludables (Generalitat de Catalunya 1984).

Lo que se espera del profesorado es que sepa actualizar el tratamiento de estos temas, evitando presentarlos únicamente desde una perspectiva médica e individualista, agregándoles la carga medioambiental que poseen. 
Esto requiere hacer intervenir a la familia, analizar con los alumnos las influencias que en sus propios comportamientos posee el entorno donde viven, desde las amistades a la estructura sanitaria, pasando por el tipo de ciudad en la que habitan o la sociedad de consumo en la que están inmersos.
Lo importante es la promoción de la salud y debemos comenzar a conseguir, desde la escuela, que las opciones de vida más fáciles de elegir sean también las más saludables.

Esquema de las fuentes sociológicas, epistemológicas, psicológicas y pedagógicas de la alimentación y nutrición

- Cultura y tradiciones

- Leyes de mercado

- Tecnología y desarrollo

- Excedentes y desperdicios

- Solidaridad

- Publicidad

- Necesidad de una correcta

alimentacion de los ciudadanos

- Historia de la alimentación

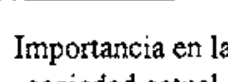

Conocimientos

científicos y su

evolucion

- Nutrición y alimentación. Concepto y necesidad

- Nutrientes y alimentos. Procedencia

- Alimentación equilibrada

- Anatomía de los aparatos digestivos

- El proceso de la digestión

- Necesidades nutricionales. Alimentación equilibrada

- Consecuencias de una alimentación incorrecta

- Higiene de los alimentos. Manipulación y conservación

- Percepciones

- Adicciones sociedad actual

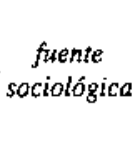

$-$

fuente pedagógica

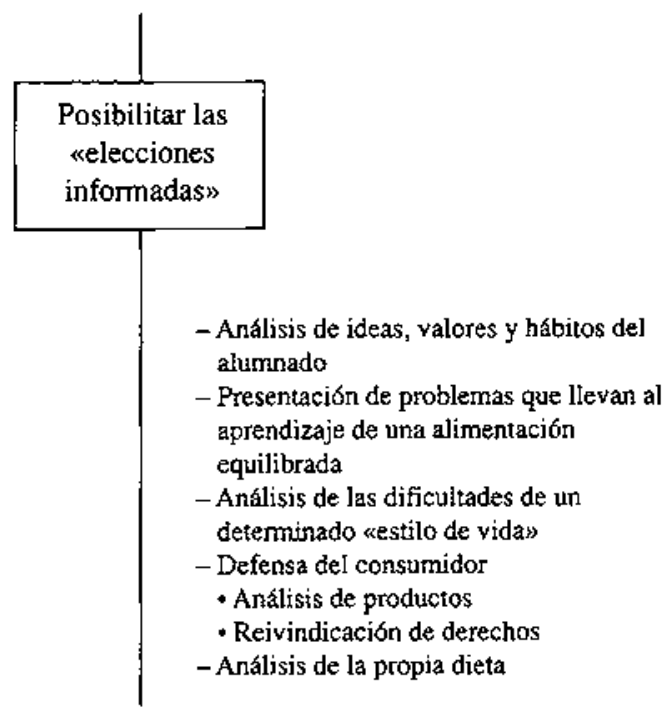

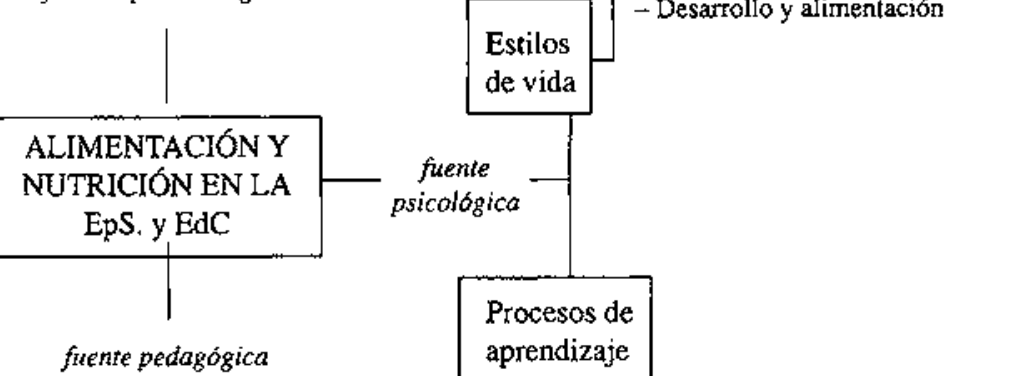

- Satisfacción de necesidades (fisiológicas, sentidas, inducidas)

- Inffuencia de la publicidad

- Alteraciones de la conducta alimentaria (anorexia, obesidad, bulimia)

- Comportamiento alimentario

fuente epistemológica 


\section{REFERENCIAS BIBLIOGRÁFICAS}

AUSUBEL, D.P., 1978. Psicología educativa. Un punto de vista cognoscitivo. (Trillas: México).

COLL,C., 1986. Marc curricular peral' ensenyamentobligatori. (Generalitat de Catalunya. Departament d'Ensenyament).

DRIVER, R., 1986. Psicología cognoscitiva y esquemas conceptuales de los alumnos, Enseñanza de las Ciencias, 4 (1) pp. 3-15.

FERRARA, F.A., ACEBAL, F. y PAGANINI, J.M., 1975. Medicina de la Comunidad. (Intermédica: Buenos Aires).

GENERALITAT DE CATALUNYA. 1984. Educació per a la salut a l'escola. Orientacions i programes. (Barcelona).

GIMENO SACRISTÁN, J., y PÉREZ, A., 1983. La enseñanza, su teoría y su práctica. (Akal: Madrid).

LATHEM, W. y NEWBERRY, A. 1970. Community medicine teaching, research and health care. (Meredith Corporation: Nueva York).

LAW, M.L., 1980. Public Health in the 80's. A Decade of Decline or an Opportunity for change, Canadian Journal of Public Health, 71, pp. 249-253.
LINVILLE, H.R., 1909. The practical use of biology, School Science and Mathematics, 9.

MODOLO, M.A., 1979. Educación Sanitaria, comportamiento y participación, $/ /$ pensiero scientifico, compilación CAPS, 8, pp. 39-58.

NOVACK, J.D., 1988. Constructivismo humano: un consenso emergente, Enseñanza de las Ciencias, Vol. 6, pp. 213-223.

OMS, 1954. Serie de Informes Técnicos. (OMS: Ginebra).

OMS, 1981. Estrategia Mundial de Salud para todos en el año 2000. (OMS: Ginebra).

SALLERAS SANMARTf, L., 1985. Educación Sanitaria. Principios, métodos y aplicaciones. (Díez de Santos: Madrid).

SCHWARTZ, K., 1974. The theory and practice of community medicine, Public Health, 80, pp. 261-265.

TERRIS, M., 1980. La Revolución Epidemiológica y la Medicina Social. (Siglo XXI: México).

WINSLOW, C.E.A., 1923. Evolution and Significance on the Modern Public Health Campaigns. (Yale University Press: New Haven). 